\title{
Labyrinthe
}

$37 \mid 2011$ (2)

Des séries et des vies

\section{Espace Mémoire Événement (The Wire)}

\section{Elisabeth Hodges}

\section{(2) OpenEdition}

Journals

Édition électronique

URL : http://journals.openedition.org/labyrinthe/4191

DOI : $10.4000 /$ labyrinthe.4191

ISSN : 1950-6031

Éditeur

Hermann

Édition imprimée

Date de publication : 15 août 2011

ISBN : 9782705681470

Référence électronique

Elisabeth Hodges, « Espace Mémoire Événement (The Wire) », Labyrinthe [En ligne], 37| 2011 (2), mis en ligne le 01 août 2013, consulté le 30 avril 2019. URL : http://journals.openedition.org/ labyrinthe/4191; DOI : 10.4000/labyrinthe.4191 


\title{
Espace Mémoire Événement (The Wire)
}

\author{
Elisabeth Hodges \\ Contact: hodgesed@muohio.edu
}

La troisième saison de la série The Wire (en français Sur écoute, 2002$\left.2008^{1}\right)$ s'ouvre à notre regard par un léger travelling arrière qui suit à distance trois personnages, Poot, Bodie et un autre ami, qui marchent vers la caméra en parlant de la destruction imminente de la cité de Franklin Towers, le territoire qui était jusque-là le site privilégié de leur négoce de drogue à Baltimore pour le compte d'Avon Barksdale, narcotrafiquant au centre de la première saison. Alors que ces trois amis errent vers le site de cette perte imminente, une absence s'instaure dans le plan étant donnée la distance que garde la caméra qui, en dépit de cela, les fixe d'un regard mobile agencé par ce travelling arrière qui nous permet paradoxalement d'être à l'écoute, malgré la distance et le mouvement. Quoique l'on soit d'abord sensibilisé à voir, à chercher à attribuer un sens à l'image, cette séquence réaffirme combien l'univers du "Wire " nous oblige aussi à écouter attentivement, comme le suggère son titre, témoignant par l'ouïe et non seulement par la vision. La scène commence in medias res, surprenant la trajectoire de ces trois amis tout en captant des bribes de conversation saisis grâce à cette caméra qui nous permet d'organiser l'accès à un univers dès lors quasi-inconnu par le téléspectateur américain, plus habitué à disparaître dans le monde préalable et artificiel de la reality $T V$. Il s'agit ainsi d'un privilège mixte - une chance, mais aussi une expérience d'arrachement à son univers familier - d'aborder le réalisme tragique des vies de ces jeunes ambitieux qui ne veulent qu'ériger leur place dans un monde qui leur refuse son accès soit à cause d'un non-dit, le racisme, soit à cause de leur situation sociale.

1. La troisième saison correspond à une diffusion en 2004-2005. Pour plus de renseignements sur la série, voir l'entrée dans l'Internet Movie Database, http://www.imdb.com/title/tt0306414. La séquence elle-même peut être visionnée à cette adresse: http://www.youtube.com/watch?v=pbK5HIfdyWc. 


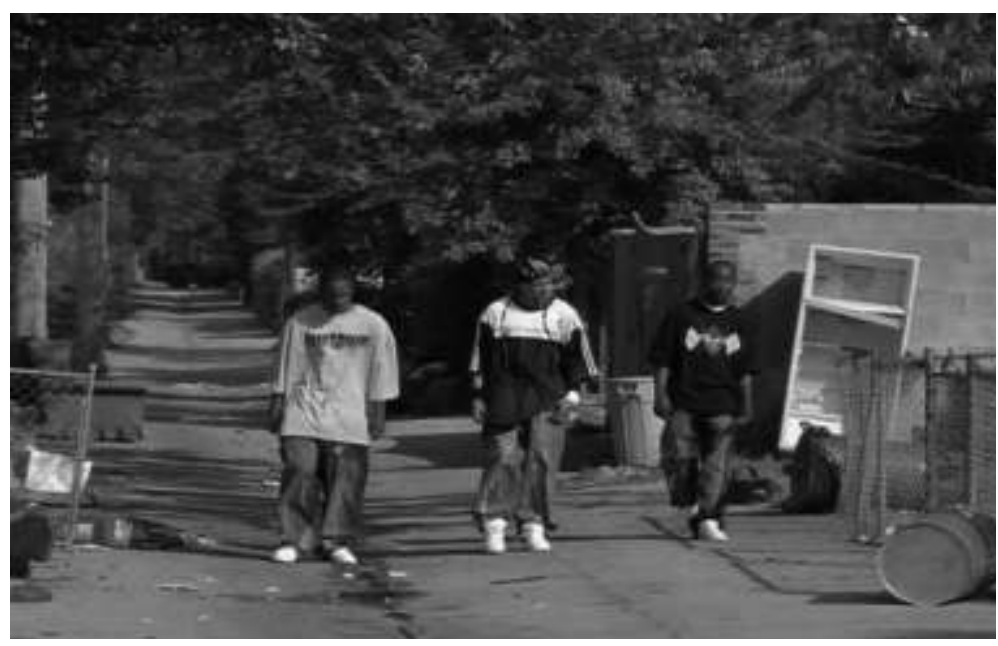

La gravité de l'événement à venir, cet effacement d'un espace qui fonctionnait en tant que lieu de mémoire à la fois collective et individuelle, semble diriger les personnages vers un avenir pour une fois certain: la fin d'un monde, de leur monde à eux. La caméra s'écarte de l'ordre prescrit via le réseau présumé logique de ces petites allées qui organisent le labyrinthe urbain dans cette partie de la ville, fournissant à ces jeunes hors-la-loi un endroit où échapper à la police (appelée : la «popo »). En même temps que les trois amis avancent, ils restent toujours visibles pour le spectateur, centrés dans un cadre mobile qui ne les quitte pas de vue. Ils ne semblent pas résister à la force presque centrifuge de la destruction de la cité, cet espace qui abritait leurs espoirs et leurs désirs; au contraire, ils marchent à grands pas vers la droite de l'écran, vers la fatalité de cet événement, afin de se joindre à la foule venue pour témoigner du moment. Ils y marchent comme guidés par la force même du destin, entourés aussi par les vestiges symboliques du monde qui est le leur - des déchets laissés par terre, une étagère cassée et abandonnée, des voitures saccagées et tout ceci cerné par des barrières de grillage métallique qui ne peuvent ni contenir les poubelles jetées par ci par là, ni préserver la frontière entre la propriété privée et l'errance de ces jeunes qui appartiennent à la ville elle-même. Ils avancent vers la foule rassemblée pour témoigner du spectacle à venir, venue comme si elle était là pour voir disparaître ces tours, symboles du mal qu'il fallait 
effacer et de l'espace et de la mémoire collective. Ce qui les attend, juste en dehors de l'espace diégétique est cet événement vers lequel se dirige le regard de la caméra qui délimite selon la logique du champ/ contre-champ ces deux points de vue impossibles à réconcilier en réalité, reliant paradoxalement les deux espaces mis en dialogue par la double perspective de la caméra; point de vue qui imite notre propre rencontre avec le monde aliénant avec lequel nous sommes confrontés dès les premiers plans de la série.

À travers les prises de vue comme celle-ci s'inaugure la vision de l'univers de David Simon (créateur de la série), vision qui voudrait servir de métaphore pour la troisième saison. Comme Simon l'explique dans le commentaire DVD, le grand angle, et bas, de la caméra permet d'englober l'espace urbain, d'offrir à l'œil et à l'écoute du spectateur attentif ces rencontres avec un monde qui nous est totalement étranger ${ }^{2}$. Quoiqu'on reconnaisse facilement les images malheureusement trop familières des quartiers défavorisés ainsi que l'urbanisme coupable d'avoir créé ces cités dans les années soixante aux États-Unis comme en France (où l'objectif semble d'y endiguer la pauvreté afin de l'effacer de notre vue), c'est davantage la langue quasi incompréhensible parsemée des codes inventés pour détourner la police qui nous désoriente dans ces paysages urbains $^{3}$. Le monde que Simon nous fait découvrir paraît coïncider avec les images médiatiques (et souvent caricaturales) de ces environnements trop souvent représentés dans les médias pour la délinquance qui y a lieu, mais c'est précisément dans la distance entre la caricature et l'hyper-réalisme de la caméra qu'opère le génie de Simon: tout en nous désorientant, il

\footnotetext{
2. À voir l'entretien de Nick Hornby avec David Simon paru dans The Believer, Vol. 5, no. 6 (août 2007) dans lequel Simon déclare à propos du public et de son œuvre: «My standard for verisimilitude is simple and I came to it when I started to write prose narrative: fuck the average reader. [...] He knows nothing and he needs everything explained to him right away, so that exposition becomes this incredible story-killing burden. Fuck him. Fuck him to hell.» (p. 76-77) (« Mon principe pour la vraisemblance est simple et je l'ai établi quand j'ai commencé à écrire des récits en prose: que le lecteur moyen aille se faire foutre. [...] Il ne sait rien et voudrait que tout lui soit expliqué tout de suite, de sorte que l'introduction devient cet incroyable fardeau qui tue l'histoire. Qu'il aille se faire foutre. Qu'il aille se faire foutre en enfer. »)

3. La critique aigue de cet urbanisme me semble lier The Wire à des films comme La Haine de Mathieu Kassovitz (1995) qui paraissent interroger la politique derrière la construction de ces ghettos. A voir l'article de Ginette Vincendeau « Designs on the Banlieue: Mathieu Kassovitz's 'La Haine' (1995)» dans Susan Hayward et Ginette Vincendeau, éds., French Film : Texts and Contexts (London: Routledge, 2000): $310-327$.
} 
nous rapproche paradoxalement de ce monde à travers cette éthique qui s'instaure par le fait d'être à l'écoute de l'autre.

Comme l'indique le titre, on est censé non seulement voir attentivement mais aussi être à l'écoute de ce qui se passe à l'écran, témoignant d'une visibilité qui ne se donne à voir qu'à partir du moment où la voix rejoint et explique l'image en une harmonie presque parfaite. Tout comme les souvenirs dont s'entretiennent Poot et Bodie dans ce plan-séquence, l'acte de la mémoire s'accomplit dans le lien qu'ils érigent entre vision et parole: "Seen some shit in them Towers that still make me smile» ( «J'ai vu des choses dans ces tours qui me font toujours sourire $»^{4}$ ). Étrange détour que prend Simon ici, alors qu'on aurait plutôt anticipé un dialogue semé par le regret et la fatalité destructrice de la transformation que revendique le maire. Au contraire, il fait coïncider chez les personnages la perte de leur monde avec le plaisir des souvenirs volatiles qui, quoiqu'attachés au lieu où les événements se sont passés, échappent néanmoins à l'immobilité immanente d'un espace qui dès lors n'aura sa place que dans la mémoire poussiéreuse de ceux qui y ont vécu:

- Poot: «I'm talking about people, memories, and shit. » (« Je parle des gens, des souvenirs et d'autres conneries »)

- Bodie: "That ain't the same. They gonna tear this building down and build some new shit. But people? They don't give a fuck about people. » («C'est pas la même chose. Ils vont raser cette tour et construire une nouvelle connerie/merde. Mais les gens? Ils n'en ont rien à foutre des gens »)

Face à l'urbs fragile, vulnérable, à la démarche de la réforme politique qui, en rasant la tour, voudrait ériger une nouvelle Baltimore à sa place, Bodie revendique l'élément qui manque aux projets des réformateurs (cible de la plume âpre de la troisième saison): la civitas, le peuple sans qui il n'y aurait ni ville, ni société, ni mémoire. Mais tout comme les déchets qui encadrent ces héros improbables dans le premier plan de la séquence, la réforme ne s'adresse qu'à l'espace, qu'à la surface du mal que le maire cherche à éradiquer de la mémoire collective, oubliant

4. Toutes les traductions du dialogue sont miennes ainsi que les maladresses. Il m'était impossible de ne pas trahir la spécificité linguistique de leur langue, déjà assez difficile à comprendre en anglais, j'ai donc choisi plutôt de rendre le sens général en français. 
ceux qui l'agencent et y vivent - faisant du lieu un espace qui leur est propre $^{5}$. Dans un geste de substitution métonymique, Poot associe la perte tragique de la tour avec une autre, celle de sa virginité, mêlant au plaisir de l'eros, comme si le pouvoir de ces souvenirs pourrait différer la fin, le thanatos de cet espace devenu leur monde à eux - comme si la volupté qui s'y trouvait devait résister à l'effondrement de ces bâtiments qui abritent toute l'histoire de leur monde.

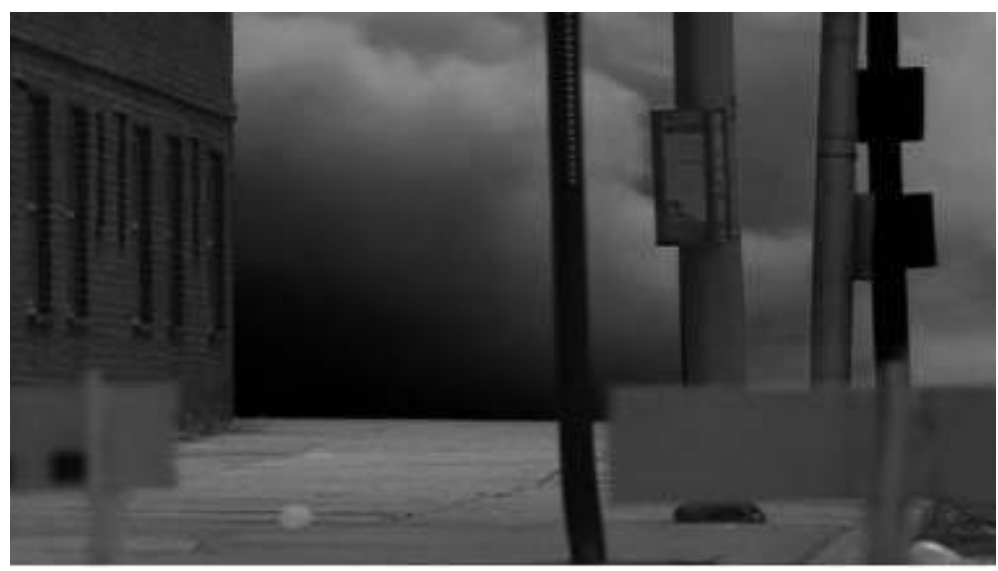

Il s'agit, à mon avis, d'un espace marqué par un dispositif de disparition qui anticipe l'effacement à venir (l'immolation de la tour) et ainsi du lieu de résistance qui procurait à ces personnages un espace qui leur était propre. Même dans les contours répétitifs et inhumains de l'architecture fonctionnelle de la cité de Franklin Towers que l'on voit de loin pendant quelques secondes juste avant sa destruction, Poot et Bodie ne voient pas un espace homogène ou aliénant, bien au contraire. Pour eux,

5. Dans L'Invention du quotidien. Tome I: Arts de faire, Paris, Gallimard « Folio », 1980, Michel de Certeau distingue la notion de lieu (espace schématique) de celle de l'espace (activée par l'intervention de la pratique). 
l'immeuble est un lieu de mémoire qui, même après sa disparition, durera autant qu'ils pourront transmettre leur savoir de cet espace de bouche à oreille, avec le plaisir de la parole. Même si dans le cadre de l'urbanisme des grandes villes américaines telles que Baltimore, le plan participe à une logique organisatrice faisant partie du système institutionnel et spatial qui a pour but de contenir le « mal » invisible du siècle qui est le nôtre (la pauvreté) et que cible l'œil et la plume critique de David Simon à travers les cinq saisons de la série; la police, les dockers, la réforme, l'éducation et la presse, ces habitants arrivent à déployer une forme de liberté dans un espace dessiné, si l'on peut dire, pour les contenir ${ }^{6}$. Car la ville ne se compose pas uniquement de béton et de ciment, comme le note Bodie, c'est un réseau complexe constitué des relations qui s'y érigent entre l'espace, la mémoire et le désir que le pouvoir ne saurait circonscrire.

La tentative de contrôler les individus à travers l'organisation de l'espace urbain se révèle ainsi un échec lorsqu'à la fin de la séquence l'effondrement de la tour, en même temps que les héros de la série qui savent résister aux institutions corrompues, obscurcit littéralement la vision idéalisée du maire pour une « nouvelle » Baltimore.

Un nuage de poussière envahit rapidement les rues, traversant le plan de gauche à droite - la même direction prise par les trois amis avec qui la séquence a commencé - joignant ainsi le début et la fin et les deux éléments, humain et matériel: les trois héros avec l'espace tragique de la tour qui tombe en morceaux et se répand en cendres dans le paysage urbain, à la grande surprise du maire et des spectateurs qui semblent s'étonner de cet effet inattendu de l'explosion. Référence visuelle qu'il est impossible, après le 11 septembre, de ne pas voir comme un écho aux images traumatisantes de la perte de deux autres tours - images qui font partie depuis de notre mémoire collective.

En incorporant la tour dans la ville, Simon semble fixer son regard critique non sur les causes du mal que les politiciens de la troisième saison cherchent à transformer, mais sur la gravité des effets qui, sous l'œil menaçant du feu rouge du dernier plan, semble mettre fin à toute tentative de réforme avant même que ne débute l'épisode. Feu qui est légèrement plus visible et qui se trouve juste devant une autre tour

6. Voir l'article d'Emmanuel Burdeau «Qu'à un fil. Sur écoute/The Wire » Cahiers du cinéma, mars 2007, p. 74-76. 


\section{Espace Mémoire Événement (The Wire)}

hérissée de flèches cruciformes - symbole de l'institution religieuse qui n'a guère de présence dans The Wire, si ce n'est qu'elle semble souligner l'effet à la fois onirique et apocalyptique de ce dernier regard avec lequel commence la troisième saison.




